\title{
Optimized DFT-FB Transceivers over LTV Channels
}

\author{
Chih-Hao Liu and P. P. Vaidyanathan \\ Dept. of Electrical Engineering, MC 136-93 \\ California Institute of Technology, Pasadena, CA 91125, USA \\ E-mail: chliu@caltech.edu and ppvnath@systems.caltech.edu
}

\begin{abstract}
In this work, we consider the optimization of DFT modulated filterbank transceiver (DFT-FBT) over linear time varying (LTV) channels. The DFT-FBT is a generalization of the Affine Fourier transform based OFDM (Affine OFDM) and the chirped OFDM, which are suggested in recent literature for the transmission over LTV channels. For both known LTV channels and unknown wide sense stationary uncorrelated scattering (WSSUS) statistical channels, we show how to optimize the transmitting and receiving prototypes of DFT-FBT such that the signal to interference and noise ratio (SINR) at the receiver is maximized. After the optimization, the channel dependent part, like OFDM, is a set of scalar multipliers at the receiver end that adapts to the equivalent memoryless channel on a block basis. Simulation results show that the bit error rate (BER) performance of the optimized DFT-FBT over LTV channels is superior to the Affine OFDM.

Index Terms - OFDM, Doubly-Selective channel, chirped
\end{abstract} OFDM, DFT FB Transceiver, wireless multicarrier systems.

\section{INTRODUCTION}

In recent years, orthogonal frequency division multiplexing (OFDM) systems have found many applications in wideband communications. One of the advantages of OFDM systems is their ability to combat ISI induced by the transmission over frequency selective channels efficiently. Due to its many fascinating features, OFDM technology has been adopted for the wireless wideband communications systems, like DVB$\mathrm{T}$ [7] and the envolving IEEE 802.16e standard for WiMAX [8]. In these applications, the wireless channels are typically LTV multipath channels, which are also called doubly selective channels. The time variation may be caused by Doppler shift due to the mobility of the transmitter or receiver, carrier frequency offset, or phase noise [4], [9]. This leads us to study the case where the channel changes continuously within one block time. Windowed Fourier functions in $\mathbf{L}^{2}\left(\mathbb{R}^{2}\right)$ [12] have been reported in [10], [11], serving as good approximate eigenfunctions of practical LTV channels. They are in the form of $f(n-l N) \exp (j 2 \pi k n / M)$. And the transceiver based on these eigenfunctions can be represented as a DFT-FBT as in Fig. 1.

Moreover, the results reported in [2], [3] also confirm this approximation. They show that if the support of the spreading function $S(v, \tau)$ of the LTV channels is maximally concentrated on a line in delay-Doppler plane, the chrip modulated fourier basis is a set of eigenfunctions of these channels. The chirped OFDM schemes in [1], [2] are generalized by [3] as Affine OFDM. However, they all fail to diagonalize general LTV channels in which the support is not necessarily on a line in delay-Doppler plane. The authors propose algorithms to optimize the chirp rate parameters at the transmitter and receiver to minimize MSE or maximize SIR at the receiver. But their algorithms are highly nonlinear and the perfect knowledge of LTV channels is required.

In this paper, we consider the general DFT-FBT whose prototypes are arbitrary and not limited to the form of chirp

${ }^{1}$ Work supported in parts by the ONR grant N00014-08-1-0709 and the California Institute of Technology waveforms as in Affine OFDM. Since the average receiving SINR has direct impact on BER performance, our goal is to optimize DFT-FBT over LTV channels such that the SINR is maximized at the receiving detectors. With the treatment of inter-symbol interference (ISI) and inter-carrier interference (ICI) as gaussian noise as in multiuser detection for CDMA systems [5], the equivalent channel is memoryless within one block time. Standard channel estimation techniques of OFDM can be applied directly to design the FEQ [6] to correct scalar ambiguity from block to block. Firstly, the case in which the transmitter and receiver have perfect knowledge of the channel is considered. We extend the SIR maximization algorithm for DFT-FBT over LTI channels [13] to our case where the channel is LTV. We show how to optimize the transmitter and the receiver such that SIR is maximized. In addition, a new iterative algorithm that can maximize SINR of DFT-FBT is proposed. Secondly, WSSUS stochastic channels [4] are considered. Based on the statistics, we propose an algorithm that optimizes the prototypes for the maximizations of average SIR/SINR. In this case, both prototypes are stationary for fixed statistics.

\section{THE FBT FOR LTV CHANNELS}

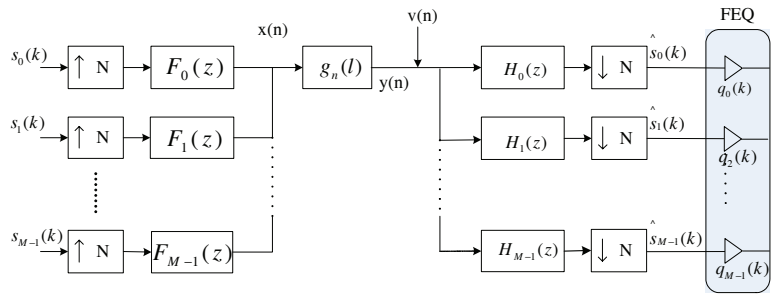

Fig. 1. General filter bank transceiver

The block diagram of a FB transceiver is shown in Fig. 1. There are $M$ subchannels and the decimation ratio is $N$. We assume that $L=N-M$ redundant samples are added, where $N>M$. The LTV channel is characterized by the channel response $g_{n}(l)$, for $0 \leq l \leq L$. In the following discussions, we will derive the input-output (I/O) description of a FBT over LTV channels. Assume that all transmitting filters $f_{i}(n)$ 's and and receiving filters $h_{i}(n)$ are of order $n_{f}$ and $n_{h}$, respectively. Let

$$
\tilde{g}_{i}^{(k)}(n)=\sum_{l=0}^{L} g_{n}(l) f_{i}[n-k N-l],
$$

which has support on $n \in k N, k N+1, \ldots, k N+n_{f}+L$. The output signal of the channel is

$$
y(n)=\sum_{k=-\infty}^{\infty} \sum_{i=0}^{M-1} s_{i}(k) \tilde{g}_{i}^{(k)}(n) .
$$


The downsampled signal after the $m$-th subband filter $H_{m}(z)$ can be expressed in Z-domain as

$$
\hat{S}_{m}(z)=\sum_{k=-\infty}^{\infty} \sum_{i=0}^{M-1} s_{i}(k)\left[H_{m}(z) \tilde{G}_{i}^{(k)}(z)\right]_{\downarrow N},
$$

in which $\tilde{G}_{i}^{(k)}(z)=\sum_{n=-\infty}^{\infty} \tilde{g}_{i}^{(k)}(n) z^{-n}$. Define

$$
g_{i}^{(k)}(n)=\tilde{g}_{i}^{(k)}(n+k N), \text { for } n \in 0,1, \ldots, n_{f}+L .
$$

With $z_{k, i}(n)$ denoting the contribution of $s_{i}(k)$ to $\hat{S}_{m}(z)$, the advanced version $z_{k, i}(n+k)$ has Z-transform

$$
s_{i}(k)\left[H_{m}(z) G_{i}^{(k)}(z)\right]_{\downarrow N} .
$$

To facilitate the derivation of I/O description, we define

$$
\left[H_{m}(z) G_{i}^{(k)}(z)\right]_{\downarrow N}=\sum_{n} b_{i, m}^{(k)}(n) z^{-n} .
$$

So, the output signal $\hat{s}_{m}(k)$ can be expressed as

$$
\begin{aligned}
\hat{s}_{m}(k)= & s_{m}(k) b_{m, m}^{(k)}(0)+\sum_{n \neq 0} s_{m}(k-n) b_{m, m}^{(k-n)}(n) \\
& +\sum_{i \neq m} \sum_{n} s_{i}(k-n) b_{i, m}^{(k-n)}(n) .
\end{aligned}
$$

From the above expression, we have the time-varying I/O description for the FBT from the $i$-th input to the $m$-th output at the $k$-th block time as

$$
T_{i, m}^{(k)}(z)=b_{i, m}^{(k)}(0)+\sum_{n \neq 0} b_{i, m}^{(k-n)}(n) z^{-n}
$$

For the $m$-th output, the ISI and ICI come from $\sum_{n \neq 0} b_{m, m}^{(k-n)}(n) z^{-n}$ and $\sum_{n} b_{i, m}^{(k-n)}(n) z^{-n}$, respectively, for all $i \neq m$. Note that $T_{i, m}^{(k)}(z)$ is a useful notation, although there is no physical system with this transfer function. Unfortunately, the ISI-free solution for LTV channels is unknown. To have near ISI-free property, the transmitting filters $F_{i}(z)$ and the receiving filters $H_{i}(z)$ could be optimized such that these terms are as small as possible.

For efficient implementation, the class of DFT-FBT is often considered a good candidate. The transmitting filters $F_{i}(z)$ and the receiving filters $H_{i}(z)$, for $0 \leq i \leq M-1$, are modulated versions of prototype filters $h(n)$ and $f(n)$ given by

$$
\begin{aligned}
& F_{i}(z)=W_{M}^{i L} \sum_{n=0}^{n_{f}} f(n) W_{M}^{-i n} z^{-n} \\
& H_{i}(z)=W_{M}^{-i L} \sum_{n=0}^{n_{h}} h(n) W_{M}^{i n} z^{n}
\end{aligned}
$$

where $W_{M}=\exp (-j 2 \pi / M)$.

The special case of DFT-FBT with $n_{f}=n_{h}=N-1$ is as in Fig. 2. It becomes the Affine OFDM if $h(0)=\ldots=$ $h(L-1)=0$, and

$$
\begin{aligned}
& f(n)=e^{j 2 \pi c_{1}(n-L)^{2}}, \\
& h(n)=e^{j 2 \pi\left(c_{0}(n-L)-c_{1}(n-L)^{2}\right)},
\end{aligned}
$$

in which $c_{0}$ and $c_{1}$ are constant. The authors in [3] show that if the LTV channel takes the form of

$$
g_{n}(l)=v(l) e^{-j 2 \pi c_{1} l^{2}} e^{j 2 \pi\left(2 c_{1} l-c_{0}\right) n},
$$

where $v(l)$ is a function of integer $l$, the coefficients $h(n)$ and $f(n)$ would make the equivalent channel matrix from $\underline{\mathbf{s}}(k)$ to $\underline{\hat{\mathbf{s}}}(k)$ a circulant matrix. Hence, the LTV channel is diagonalizable by $\mathbf{W}$ and $\mathbf{W}^{\dagger}$. This is called linear delay/Doppler spreading channel in [2].

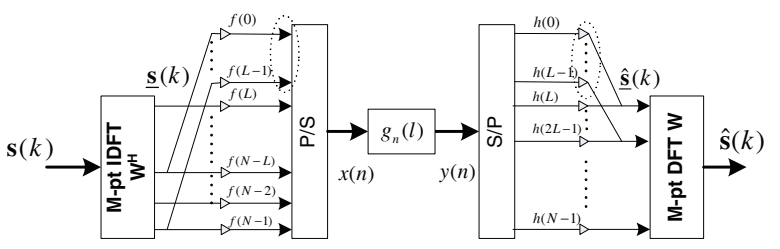

Fig. 2. DFT-FBT transceiver with $n_{h}=n_{f}=N-1$

\section{SIR OPTIMIZATION OF DFT-FBT FOR KNOWN LTV CHANNELS}

For the class of linear delay/Doppler spreading channel, the channel is always diagonalized, if the prototypes of a DFTFBT take the form of (7). However, LTV channels in general are not linear delay/Doppler spreading, i.e., the support of $S(v, l)$ is not necessarily on a line. Unfortunately, there is no ISI-free solution in terms of $F_{i}(z)$ and $H_{i}(z)$ for general LTV channels. In the following, we consider the class of DFT-FBT and show how to design the prototype filters so that SIR at receiver is maximized.

In the following discussions, the input signals $s_{i}(n)$ are assumed to be zero mean WSS and white random process with the same variance, i.e,

$$
\mathbb{E}\left\{s_{i}\left(n^{\prime}\right) s_{j}^{*}(n)\right\}=\sigma_{s}^{2} \delta(i-j) \delta\left(n^{\prime}-n\right) .
$$

Assume that the prototypes adapt to the channel on a per block basis. Let $\mathbf{f}^{(k)}=\left[f^{(k)}(0) f^{(k)}(1) \ldots f^{(k)}\left(n_{f}\right)\right]^{T}$ and $\mathbf{h}^{(k)}=\left[h^{(k)}(0) h^{(k)}(1) \ldots h^{(k)}\left(n_{h}\right)\right]^{T}$ be the transmitting and receiving prototype, respectively, at the $k$-th block time. Also, let $n_{h}$ and $n_{f}$ be no greater than $N-1$.

Define a $1 \times\left(n_{h}+1\right)$ row vector and a $1 \times\left(n_{f}+L+1\right)$ row vector as

$$
\begin{aligned}
& \mathbf{b}_{i, f}^{(k)}(n)=\left[\begin{array}{lll}
g_{i}^{(k)}(n N) & \ldots & g_{i}^{(k)}\left(n_{h}+n N\right)
\end{array}\right] \\
& \mathbf{b}_{i, h}^{(k)}(n)=\left[\begin{array}{llll}
h_{i}^{(k)}(-n N) & \ldots & h_{i}^{(k)}\left(n_{f}+L-1-n N\right)
\end{array}\right]
\end{aligned}
$$

where $h_{i}^{(k)}(\cdot)$ is the $i$-th receiving filter at the $k$-th block time. So, $b_{i, m}^{(k)}(n)$ can be expressed as the product of matrices as

$$
b_{i, m}^{(k)}(n)=\mathbf{b}_{i, f}^{(k)}(n) \mathbf{D}_{m} \mathbf{h}^{(k)}=\mathbf{b}_{m, h}^{(k)}(n) \mathbf{G}^{(k)} \mathbf{D}_{i}^{\prime} \mathbf{f}^{(k)},
$$

where

$$
\begin{gathered}
\mathbf{D}_{i}=\operatorname{diag}\left(1, W_{M}^{i}, \ldots, W_{M}^{i n_{h}}\right) W_{M}^{-i L} \\
\mathbf{D}^{\prime}{ }_{i}=\operatorname{diag}\left(1, W_{M}^{-i}, \ldots, W_{M}^{-i n_{f}}\right) W_{M}^{i L}, \\
\mathbf{G}^{(k)}=\left[\begin{array}{cccc}
g_{k N}(0) & 0 & \cdots & 0 \\
\vdots & g_{k N+1}(0) & \ddots & \vdots \\
g_{k N+L}(L) & \vdots & \ddots & 0 \\
0 & g_{k N+L+1}(L) & & g_{k N+n f}(0) \\
\vdots & & \ddots & \vdots \\
0 & \ldots & 0 & g_{k N+L+n f}(L)
\end{array}\right] .
\end{gathered}
$$


The power gains for the desired signal, the ISI and the ICI of the $m$-th output $\hat{s}_{m}(k)$ can be shown to be

$$
\begin{aligned}
\frac{P_{s i g}^{(k)}(m)}{\sigma_{s}^{2}} & =\left|b_{m, m}^{(k)}(0)\right|^{2} \\
& =\mathbf{h}^{(k) \dagger} \mathbf{D}_{m}^{\dagger} \mathbf{b}_{m, f}^{(k) \dagger}(0) \mathbf{b}_{m, f}^{(k)}(0) \mathbf{D}_{m} \mathbf{h}^{(k)} \\
\frac{P_{i s i}^{(k)}(m)}{\sigma_{s}^{2}} & =\sum_{n \neq 0}\left|b_{m, m}^{(k-n)}(n)\right|^{2} \\
& =\sum_{n \neq 0} \mathbf{h}^{(k-n) \dagger} \mathbf{D}_{m}^{\dagger} \mathbf{b}_{m, f}^{(k-n) \dagger}(n) \mathbf{b}_{m, f}^{(k-n)}(n) \mathbf{D}_{m} \mathbf{h}^{(k-n)} \\
\frac{P_{i c i}^{(k)}(m)}{\sigma_{s}^{2}} & =\sum_{n, i \neq m}\left|b_{i, m}^{(k-n)}(n)\right|^{2} \\
& =\sum_{i \neq m, n} \mathbf{h}^{(k-n) \dagger} \mathbf{D}_{m}^{\dagger} \mathbf{b}_{i, f}^{(k-n) \dagger}(n) \mathbf{b}_{i, f}^{(k-n)}(n) \mathbf{D}_{m} \mathbf{h}^{(k-n)} .
\end{aligned}
$$

Therefore, the SIR at the $k$-th block time can be expressed as

$$
S I R_{\mathbf{h}}^{(k)}=\frac{\mathbf{h}^{(k) \dagger} \mathbf{Q}_{0, f}^{(k)} \mathbf{h}^{(k)}}{\mathbf{h}^{(k) \dagger} \mathbf{Q}_{1, f}^{(k)} \mathbf{h}^{(k)}+\sum_{n \neq 0} \mathbf{h}^{(k-n) \dagger} \mathbf{Q}_{2, f}^{(k-n)}(n) \mathbf{h}^{(k-n)}}
$$

where $\mathbf{Q}_{0, f}^{(k)}, \mathbf{Q}_{1, f}^{(k-n)}$ and $\mathbf{Q}_{2, f}^{(k-n)}$ are positive definite matrices given by

$$
\begin{aligned}
\mathbf{Q}_{0, f}^{(k)} & =\sigma_{s}^{2} \sum_{m} \mathbf{D}_{m}^{\dagger} \mathbf{b}_{m, f}^{(k) \dagger}(0) \mathbf{b}_{m, f}^{(k)}(0) \mathbf{D}_{m} \\
\mathbf{Q}_{1, f}^{(k)} & =\sigma_{s}^{2} \sum_{m, i \neq m} \mathbf{D}_{m}^{\dagger} \mathbf{b}_{i, f}^{(k) \dagger}(0) \mathbf{b}_{i, f}^{(k)}(0) \mathbf{D}_{m} \\
\mathbf{Q}_{2, f}^{(k)}(n) & =\sigma_{s}^{2} \sum_{m, i} \mathbf{D}_{m}^{\dagger} \mathbf{b}_{i, f}^{(k) \dagger}(n) \mathbf{b}_{i, f}^{(k)}(n) \mathbf{D}_{m}
\end{aligned}
$$

Similarly we can express the same SIR in terms of $\mathbf{f}^{(k)}$ as

$$
S I R_{\mathbf{f}}^{(k)}=\frac{\mathbf{f}^{(k) \dagger} \mathbf{Q}_{0, h}^{(k)} \mathbf{f}^{(k)}}{\mathbf{f}^{(k) \dagger} \mathbf{Q}_{1, h}^{(k)} \mathbf{f}^{(k)}+\sum_{n \neq 0} \mathbf{f}^{(k-n) \dagger} \mathbf{Q}_{2, h}^{(k-n)}(n) \mathbf{f}^{(k-n)}},
$$

where $\mathbf{Q}_{0, h}^{(k)}, \mathbf{Q}_{1, h}^{(k)}$ and $\mathbf{Q}_{2, h}^{(k)}(n)$ are positive definite matrices similar to $\mathbf{Q}_{0, f}^{(k)}, \mathbf{Q}_{1, f}^{(k)}$ and $\mathbf{Q}_{2, f}^{(k)}(n)$ by replacing $\mathbf{b}_{m, f}^{(k)}(n)$ and $\mathbf{D}_{m}$ with $\mathbf{b}_{m, h}^{(k)}(n)$ and $\mathbf{G}^{(k)} \mathbf{D}^{\prime}{ }_{m}$, respectively.

$S I R^{(k)}$ depends on the prototypes prior to block $k$. It is not easy to optimize $S I R^{(k)}$ with respect to $\mathbf{h}^{(k)}$ or $\mathbf{f}^{(k)}$. Observe that if the first $L$ coefficients of $\mathbf{h}^{(k)}$ 's are set to zero, the interblock interference (IBI) $\sum_{n \neq 0} \mathbf{h}^{(k-n) \dagger} \mathbf{Q}_{2, f}^{(k-n)}(n) \mathbf{h}^{(k-n)}$ is zero. Hence, we assume that all the blocks are friendly blocks such that they do not allow too much IBI to leak to the other blocks. Suppose that the IBI is much smaller than the ICI within the block itself, i.e.,

$$
\begin{aligned}
\mathbf{h}^{(k) \dagger} \mathbf{Q}_{1, f}^{(k)} \mathbf{h}^{(k)} & \gg \sum_{n \neq 0} \mathbf{h}^{(k-n) \dagger} \mathbf{Q}_{2, f}^{(k-n)}(n) \mathbf{h}^{(k-n)} \\
\mathbf{h}^{(k) \dagger} \mathbf{Q}_{1, f}^{(k)} \mathbf{h}^{(k)} & \gg \sum_{n \neq 0} \mathbf{h}^{(k) \dagger} \mathbf{Q}_{2, f}^{(k)}(n) \mathbf{h}^{(k)}
\end{aligned}
$$

So,

$$
S I R_{\mathbf{h}}^{(k)} \approx \frac{\mathbf{h}^{(k) \dagger} \mathbf{Q}_{0, f}^{(k)} \mathbf{h}^{(k)}}{\mathbf{h}^{(k) \dagger}\left(\mathbf{Q}_{1, f}^{(k)}+\sum_{n \neq 0} \mathbf{Q}_{2, f}^{(k)}(n)\right) \mathbf{h}^{(k)}}=\widehat{S I R}_{\mathbf{h}}^{(k)}
$$

The SIR maximization problem becomes

$$
\max _{\mathbf{h}^{(k)}} \frac{\mathbf{h}^{(k) \dagger} \mathbf{Q}_{0, f}^{(k)} \mathbf{h}^{(k)}}{\mathbf{h}^{(k) \dagger}\left(\mathbf{Q}_{1, f}^{(k)}+\sum_{n \neq 0} \mathbf{Q}_{2, f}^{(k)}(n)\right) \mathbf{h}^{(k)}}, \text { for all } k .
$$

Solving (13) is much simpler since $\mathbf{h}^{(k)}$ could be optimized on a per block basis. The computer experiments of the optimization problem (13) for various LTV channels also confirm the friendly block assumptions in (12). Furthermore, if the assumptions are guaranteed to hold, the actual SIR at the receiver is approximately equal to the optimal SIR value in (13).

Similarly, given $\mathbf{h}^{(k)}$ and the channel response, and under similar assumptions, the optimal $\mathbf{f}^{(k)}$ can be obtained by solving the optimization problem,

$$
\max _{\mathbf{f}^{(k)}} \frac{\mathbf{f}^{(k) \dagger} \mathbf{Q}_{0, h}^{(k)} \mathbf{f}^{(k)}}{\mathbf{f}^{(k) \dagger}\left(\mathbf{Q}_{1, h}^{(k)}+\sum_{n \neq 0} \mathbf{Q}_{2, h}^{(k)}(n)\right) \mathbf{f}^{(k)}} \text {, for all } k .
$$

Given one of the two prototype filters, we can optimize the other prototype filter so that the SIR is maximized. The optimal $\mathbf{h}^{(k)}$ and $\mathbf{f}^{(k)}$ can be obtained by solving the optimization problems, involving Rayleigh-Ritz ratio, in (13) and (14), respectively. By solving the optimization problems alternatively and iteratively, the SIR will increase monotonically.

\section{SIR OPTIMIZATION FOR UNKNOWN WSSUS CHANNELS}

For wideband wireless communications, the motion of the mobile terminal, and the variation of the surrounding objects makes channels time-varying. In the design of a wideband wireless communications system over LTV channels, channel estimation is a big challenge since the channel may change due to Doppler effect within one block time. Instead of realtime LTV channel estimation, we can design a statistically optimized DFT-FBT according to the channel statistics. In the statistically optimized DFT-FBT, the transmitting and receiving filters are stationary if the channel statistics does not change. The only part that adapts from block to block is the FEQ which is designed based on the equivalent memoryless channel.

In the following, we consider $g_{n}(l)$ as a wide sense stationary uncorrelated scattering (WSSUS) channel [4]. The crosscorrelation of the zero-mean stochastic processes $g_{n}(l)$ is given by

$$
\mathbb{E}\left\{g_{n_{1}}\left(l_{1}\right) g_{n_{2}}^{*}\left(l_{2}\right)\right\}=R_{l_{2}}\left(n_{1}-n_{2}\right) \delta\left(l_{1}-l_{2}\right),
$$

where $R_{l_{2}}(n)$ is any arbitrary function. We will propose an algorithm to optimize the prototypes $\mathbf{h}$ and $\mathbf{f}$ of the DFTFBT such that the ratio of the average signal power to the average interference power is maximized. For convenience, we assume that $n_{h}=n_{f}$ and $n_{h}$ could be greater than $N-1$ since the transmitting and receiving filters are stationary. The average signal, ISI and ICI power are calculated by taking the expectation on (11) over the random process $g_{n}(l)$. From (4) and (6), one can verify that

$$
\begin{aligned}
b_{i, m}^{(k)}(n)= & \sum_{n_{1}=0}^{n_{h}} \sum_{l=0}^{L} g_{n_{1}+(n+k) N}(l) f\left(n_{1}-l+n N\right) \\
& \cdot h\left(n_{1}\right) W_{M}^{-i\left(n_{1}+n N-l-L\right)+m\left(n_{1}-L\right)} .
\end{aligned}
$$


Based on WSSUS assumption, we can express $\mathbb{E}_{g}\left|b_{i, m}^{(k)}(n)\right|^{2}$ in terms of $\mathbf{h}$ as

$$
\mathbb{E}_{g}\left|b_{i, m}^{(k)}(n)\right|^{2}=\mathbf{h}^{\dagger} \boldsymbol{\Lambda}_{m-i}^{\dagger} \mathbf{J}_{f}(n) \boldsymbol{\Lambda}_{m-i} \mathbf{h},
$$

where

$$
\begin{aligned}
\mathbf{J}_{f}(n) & =\sum_{l=0}^{L} \boldsymbol{\Delta}_{f, l, n}^{\dagger} \mathbf{J}_{l} \boldsymbol{\Delta}_{f, l, n} \\
\boldsymbol{\Lambda}_{m} & =\operatorname{diag}\left(1, W_{M}^{m}, \ldots, W_{M}^{m n_{h}}\right) \\
\boldsymbol{\Delta}_{f, l, n} & =\operatorname{diag}\left(f(-l+n N), \ldots, f\left(n_{h}-l+n N\right)\right),
\end{aligned}
$$

and $\mathbf{J}_{l}$ is an $n_{h}+1$ by $n_{h}+1$ matrix whose $\left(n_{2}, n_{1}\right)$-th element is $R_{l}\left(n_{1}-n_{2}\right)$.

Similarly, we can express $\mathbb{E}_{g}\left|b_{i, m}^{(k)}(n)\right|^{2}$ in terms of $\mathbf{f}$ as

$$
\mathbb{E}_{g}\left|b_{i, m}^{(k)}(n)\right|^{2}=\mathbf{f}^{\dagger} \boldsymbol{\Lambda}_{m-i}^{\dagger} \mathbf{J}_{h}(n) \boldsymbol{\Lambda}_{m-i} \mathbf{f}
$$

where

$$
\begin{aligned}
\mathbf{J}_{h}(n) & =\sum_{l=0}^{L} \mathbf{S}_{-l+n N}^{\dagger} \boldsymbol{\Delta}_{h}^{\dagger} \mathbf{J}_{l} \boldsymbol{\Delta}_{h} \mathbf{S}_{-l+n N} \\
\boldsymbol{\Delta}_{h} & =\operatorname{diag}\left(h(0), h(1), \ldots, h\left(n_{h}\right)\right),
\end{aligned}
$$

and $\mathbf{S}_{m}$ is an $n_{h}+1$ by $n_{h}+1$ shifting matrix that shifts a vector up by $m$. Applying these expressions, the average signal, ISI, and ICI power can be reformulated in terms of $\mathbf{f}$ or $\mathbf{h}$ as

$$
\begin{aligned}
\bar{P}_{s i g}^{(k)}(m) & =\sigma_{s}^{2} \mathbf{h}^{\dagger} \mathbf{J}_{f}(0) \mathbf{h}=\sigma_{s}^{2} \mathbf{f}^{\dagger} \mathbf{J}_{h}(0) \mathbf{f} \\
\bar{P}_{i s i}^{(k)}(m) & =\sigma_{s}^{2} \mathbf{h}^{\dagger} \sum_{n \neq 0} \mathbf{J}_{f}(n) \mathbf{h}=\sigma_{s}^{2} \mathbf{f}^{\dagger} \sum_{n \neq 0} \mathbf{J}_{h}(n) \mathbf{f} \\
\bar{P}_{i c i}^{(k)}(m) & =\sigma_{s}^{2} \mathbf{h}^{\dagger}\left(\sum_{j=1}^{M-1} \boldsymbol{\Lambda}_{j}^{\dagger} \sum_{n} \mathbf{J}_{f}(n) \boldsymbol{\Lambda}_{j}\right) \mathbf{h} \\
& =\sigma_{s}^{2} \mathbf{f}^{\dagger}\left(\sum_{j=1}^{M-1} \boldsymbol{\Lambda}_{j}^{\dagger} \sum_{n} \mathbf{J}_{h}(n) \boldsymbol{\Lambda}_{j}\right) \mathbf{f} .
\end{aligned}
$$

Note from the above expressions that the average signal, ISI, and ICI power are constant over all subbands and block time. The power is independent of block index since both $\mathbf{h}$ and $\mathbf{f}$ are stationary over the blocks and the channel is WSSUS. The independence of power from subchannel index comes from the nature of DFT-FBT and the averaging over channels. So, we have average SIR in terms of $\mathbf{h}$ as

$$
\overline{S I R}_{\mathbf{h}}=\frac{\mathbf{h}^{\dagger} \overline{\mathbf{Q}}_{0, f} \mathbf{h}}{\mathbf{h}^{\dagger} \overline{\mathbf{Q}}_{1, f} \mathbf{h}}
$$

where $\overline{\mathbf{Q}}_{0, f}, \overline{\mathbf{Q}}_{1, f}$ of dimension $n_{h}+1$ by $n_{h}+1$ are positive definite matrices

$$
\begin{aligned}
\overline{\mathbf{Q}}_{0, f} & =\sigma_{s}^{2} \mathbf{J}_{f}(0) \\
\overline{\mathbf{Q}}_{1, f} & =\sigma_{s}^{2}\left(\sum_{m=0}^{M-1} \boldsymbol{\Lambda}_{m}^{\dagger} \sum_{n} \mathbf{J}_{f}(n) \boldsymbol{\Lambda}_{m}-\mathbf{J}_{f}(0)\right) .
\end{aligned}
$$

Similarly, in terms of $\mathbf{f}$,

$$
\overline{S I R}_{\mathbf{f}}=\frac{\mathbf{f}^{\dagger} \overline{\mathbf{Q}}_{0, h} \mathbf{f}}{\mathbf{f}^{\dagger} \overline{\mathbf{Q}}_{1, h} \mathbf{f}},
$$

where $\overline{\mathbf{Q}}_{0, h}, \overline{\mathbf{Q}}_{1, h}$ can be obtained from (19) by replacing $\mathbf{J}_{f}(n)$ by $\mathbf{J}_{h}(n)$. The optimal $\mathbf{f}$ and $\mathbf{h}$ can be obtained by solving the eigen problem involving Rayleigh-Ritz ratio of (20) and (18). We can use the iterative algorithm mentioned in the previous section to get satisfactory SIR.

\section{THE SINR OPTIMIZATION}

The BER of communications systems depends on the receiving SINR. In light of this dependence, we propose algorithms to maximize the receiving SINR in this section. Assume that the noise $v(n)$ is AWGN with zero mean and variance $\sigma_{v}^{2}$. The power of receiving noise after the receiving filter $H_{k}(z)$ can be shown to be

$$
\sigma_{v}^{2} \sum_{n=0}^{n_{h}}|h(n)|^{2}
$$

So, we can formulate the average SINR for the unknown channel case in terms of $\mathbf{h}$ as

$$
\overline{S I N R}_{\mathbf{h}}=\frac{\mathbf{h}^{\dagger} \overline{\mathbf{Q}}_{0, f} \mathbf{h}}{\mathbf{h}^{\dagger}\left(\overline{\mathbf{Q}}_{1, f}+\sigma_{v}^{2} \mathbf{I}\right) \mathbf{h}},
$$

From the last section, we know that all the subchannels have the same the average $\overline{S I N R}_{\mathrm{h}}$. So, the maximization of $\overline{S I N R}_{\mathbf{h}}$ is equivalent to the minimization of the BER of DFTFBT provided that the BER is a monotonic decreasing function of SINR. Given $\mathbf{f}$, we can solve for the optimal $\mathbf{h}$ such that SINR is maximized just as in SIR maximization. However, given $\mathbf{h}$, the SINR optimization problem is

$$
\max _{\mathbf{f}} \frac{\mathbf{f}^{\dagger} \overline{\mathbf{Q}}_{0, h} \mathbf{f}}{\mathbf{f}^{\dagger} \overline{\mathbf{Q}}_{1, h} \mathbf{f}+\sigma_{v}^{2} \mathbf{h}^{\dagger} \mathbf{h}} \text { s.t. }\|\mathbf{f}\| \leq 1 .
$$

This is not a standard SIR maximization problem and cannot be solved directly. However, by Lemma 1 below, it is equivalent to

$$
\max _{\mathbf{f}} \frac{\mathbf{f}^{\dagger} \overline{\mathbf{Q}}_{0, h} \mathbf{f}}{\mathbf{f}^{\dagger}\left(\overline{\mathbf{Q}}_{1, h}+\sigma_{v}^{2} \mathbf{h}^{\dagger} \mathbf{h}\right) \mathbf{f}} \text { s.t. }\|\mathbf{f}\|=1,
$$

where $\overline{\mathbf{Q}}_{0, h}$ and $\overline{\mathbf{Q}}_{1, h}+\sigma_{v}^{2} \mathbf{h}^{\dagger} \mathbf{h}$ are positive definite matrices. Similar technique has been used in [14]. We recognize this is the same as the SIR maximization problem in the previous section. Therefore, we can solve $\mathbf{h}$ and $\mathbf{f}$ iteratively to get a satisfactory SINR value. The SINR maximization for DFTFBT over known LTV channels can be solved similarly.

Lemma 1: The optimal solution of the optimization problem (22) is $\mathbf{f}^{* *}=\mathbf{f}^{*} /\left\|\mathbf{f}^{*}\right\|$, where $\mathbf{f}^{*}$ is the optimal solution to the unconstrained optimization problem

$$
\max _{\mathbf{f}} \frac{\mathbf{f}^{\dagger} \overline{\mathbf{Q}}_{0, h} \mathbf{f}}{\mathbf{f}^{\dagger}\left(\overline{\mathbf{Q}}_{1, h}+\sigma_{v}^{2} \mathbf{h}^{\dagger} \mathbf{h}\right) \mathbf{f}},
$$

Proof: For any nontrivial $\|\mathbf{f}\| \leq 1$, let $\mathbf{f}_{3}=\mathbf{f} /\|\mathbf{f}\|$ and $\eta=\sigma_{v}^{2} \mathbf{h}^{\dagger} \mathbf{h}>0$. The ratio

$$
\frac{\mathbf{f}^{\dagger} \overline{\mathbf{Q}}_{0, h} \mathbf{f}}{\mathbf{f}^{\dagger} \overline{\mathbf{Q}}_{1, h} \mathbf{f}+\eta}=\frac{\mathbf{f}_{\mathbf{3}}{ }^{\dagger} \overline{\mathbf{Q}}_{0, h} \mathbf{f}_{\mathbf{3}}}{\mathbf{f}_{\mathbf{3}}{ }^{\dagger} \overline{\mathbf{Q}}_{1, h} \mathbf{f}_{\mathbf{3}}+\eta /\|\mathbf{f}\|^{2}} \leq \frac{\mathbf{f}_{\mathbf{3}}{ }^{\dagger} \overline{\mathbf{Q}}_{0, h} \mathbf{f}_{\mathbf{3}}}{\mathbf{f}_{\mathbf{3}}{ }^{\dagger} \overline{\mathbf{Q}}_{1, h} \mathbf{f}_{\mathbf{3}}+\eta} .
$$

The last inequality holds if and only if $\|\mathbf{f}\|=1$. So, for the optimization problem (22), we just need to consider the feasible set $\|\mathbf{f}\|=1$, and this is equivalent to (23). And (23) is equivalent to the unconstrained problem in (24) since if $\mathbf{f}^{*}$ maximizes (24), so does $\mathbf{f}^{*} /\left\|\mathbf{f}^{*}\right\|$. 


\section{Design EXAMPles}

In this section, we design DFT-FBT over WSSUS channels and compare the performance with Affine OFDM and traditional OFDM. The the channel response $g_{n}(l)$ is a wide-sense stationary stochastic process given by

$$
g_{n}(l)=\sum_{i=0}^{K-1} \alpha_{l}(n) \delta(l-i),
$$

where $K$ is the number of multipaths and the path gains $\alpha_{l}(n)$ have zero mean and follows Clark's model [4]

$$
\mathbb{E}\left[\alpha_{l}(n) \alpha^{*}\left(n_{1}\right)\right]=P_{l} J_{0}\left(2 \pi f_{D} T_{s}\left(n-n_{1}\right)\right) .
$$

$f_{D}$ is the maximum Doppler shift depending on the mobile agent velocity $v$ and is given by $f_{D}=f_{c} v / c$, where $c$ is the speed of light and $f_{c}$ is the carrier frequency. $P_{l}$ denotes the average power of the $l$-th path and $T_{s}$ is the sampling period. Here, $f_{c}$ is set to $5 \mathrm{GHz}$ and $T_{s}$ to $2 \mu s$. The average power gain for $l$-th path is $P_{l}=2^{-l}$. The channel gains are normalized such that the sum of the power from all multipaths is one. The number of subbands is $M=64, K=9$, and the size of a block $N=80$. The length of prototype filters $\mathbf{h}$ and $\mathbf{f}$ are 80 . For the known channel case, Affine OFDM and DFT-FBT are designed based on $g_{n}(l)$. The Chirp rate parameters $c_{1}$ and $c_{0}$ in (7) of the Affine OFDM are designed and updated on a per block basis by the algorithm in [3]. We design our DFTFBT by alternatively optimizing $\mathbf{h}^{(k)}$ and $\mathbf{f}^{(k)}$ to maximize the SINR. The prototypes are also updated on a per block basis. For the unknown channel case, we design the statistical optimized DFT-FBT according to the channel statistics (Sec. IV), $P_{l}$ and $f_{D}$. For the optimization of $\mathbf{h}$ and $\mathbf{f}$, the objective function are the average SINR given in (18) and (20).

The plot of SINR at the receiver versus the mobile terminal velocity is shown in Fig. 3. The variance of AWGN noise is 0.001 . The SINR is the average SINR over 500 realizations of channels. We observe that the increase of velocity, hence the Doppler frequency, lowers the SINR at the receiver. The DFT-FBT with known channel maintains really good SINR even at high Doppler frequency, while the SINRs of the traditional OFDM and the Affine OFDM drop tremendously. Furthermore, the statistical DFT-FBT (Sec. IV) outperforms the Affine OFDM even though it only has the statistics of the LTV channels and the prototypes are stationary over all transmission blocks.

Fig. 4 shows the bit error rate performance of OFDM, Affine OFDM, DFT-FBT and statistical DFT-FBT. FEQ as in Fig. 1 is used. 4-QAM symbols are transmitted in each subband. The BER is the average probability of error over 500 realizations of channels. The velocity of the mobile terminal is $100 \mathrm{~km} / \mathrm{hr}$. The Affine OFDM outperforms traditional OFDM only when $E_{b} / N_{0}$ is greater than $15 \mathrm{~dB}$. The gain of DFTFBT and statistical DFT-FBT over Affine OFDM increases as $E_{b} / N_{0}$ gets larger. When BER equals to $3 \cdot 10^{-3}$, the gains are approximately $7 \mathrm{~dB}$ and $4 \mathrm{~dB}$, respectively.

\section{CONCLUSION}

We have proposed methods of designing DFT-FBT over known and unknown LTV channels. By formulating the SINR as a Rayleigh-Ritz ratio, we are able to optimize the prototypes of DFT-FBT such that the SINR at the receiver is maximized. Even when only channel statistics are available, the statistical DFT-FBT performs better than the Affine OFDM which requires exact channel knowledge. In general, the optimized DFT-FBT provides substantial performance gain over the Affine OFDM in LTV channels.

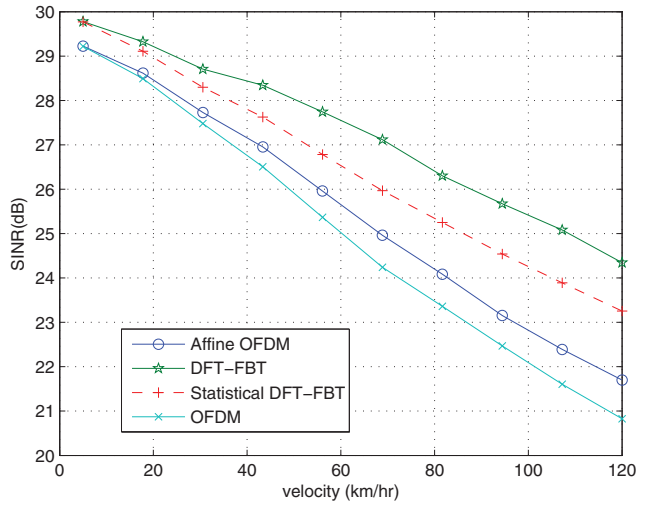

Fig. 3. Optimized SINR versus speed

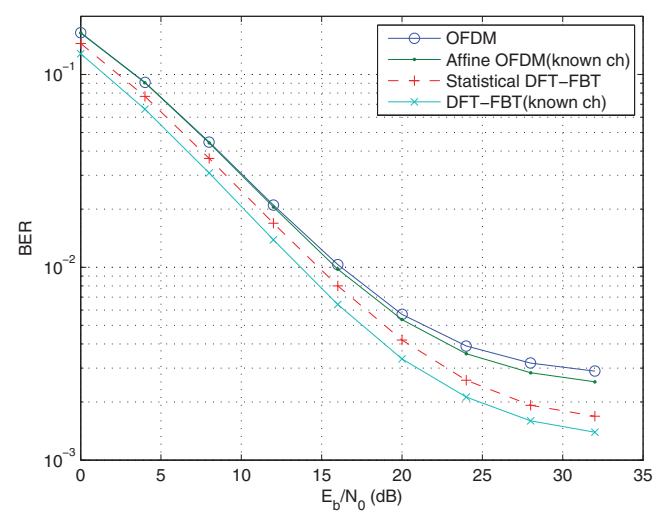

Fig. 4. Bit error rate versus $E_{b} / N_{0}$

\section{REFERENCES}

[1] M. Martone, "A Multicarrier System Based on the Fractional Fourier Transform for Time-Frequency-Selective Channels," Proc. IEEE ICASSP, May 2001.

[2] S. Barbarossa, R. Torti, "Chirped-OFDM for Transmissions over TimeVarying Channels with Linear Delay/Doppler Spreading," IEEE Trans. Communications, Vol. 49, June 2001.

[3] T. Erseghe, N. Laurenti and V. Cellini, "A Multicarrier Architecture Based upon the Affine Fourier Transform," IEEE Trans. Communications, Vol. 53, May 2005.

[4] S. Haykin and M. Moher, Modern Wireless Communications, Prentice Hall 2005.

[5] S. Verdu, Multiuser Detection, Cambridge 1998.

[6] S. Coleri, M. Ergen, A. Puri and A. Bahai, "Channel estimation techniques based on pilot arrangement in OFDM systems," IEEE Trans. Broadcasting, Vol. 48, Sep. 2002.

[7] U. Reimers, "DVB-T: the COFDM-based system for terrestrial television," Electronics and Communication Engineering Journal," Vol. 9, Feb. 1997.

[8] IEEE Std 802.16e, "Amendment for physical and medium access control layers for combined fixed and mobile operation in licensed bands," Sep. 2005.

[9] T. Pollet, M. V. Bladel and M. Moeneclaey, "BER sensitivity of OFDM systems to carrier frequency offset and Wiener phase noise," IEEE Trans. Communications, Vol. 43, April 1995.

[10] K. Liu, Y. Kadous, A. M. Sayeed, "Orthogonal Time-Frequency Signaling over Doubly Dispersive Channels," IEEE Trans. Information Theory, Vol. 50, Nov. 2004.

[11] W. Kozek and A. F. Molishch, "Nonorthogonal pulse shapes for multicarrier communications in Doubly Disprsive Channels," IEEE J. Select. Areas Communications, Vol. 16, Oct. 1998.

[12] S. Mallat, A Wavelet Tour of Signal Processing, Academic Press, 2009.

[13] S. M. Phoong, Y. B. Chang, C. Y. Chen, "DFT Modulated Filter Bank Transceivers for Multipath Fading Channels," IEEE Trans. Signal Processing, Vol. 53, No. 1, Jan. 2005.

[14] C. Y. Chen, P. P. Vaidyanathan, "MIMO Radar MIMO Radar Waveform Optimization with Prior Information of the Extended Clutter and Target," submitted to IEEE Trans. Signal Processing. 